\title{
Vehicle accident management and control system using MQTT
}

\author{
Sudha Senthilkumar, K. Brindha, Shashank Bhandari \\ School of Information Technology and Engineering, Vellore Institute of Technology, India
}

\begin{tabular}{l}
\hline \hline Article Info \\
\hline Article history: \\
Received May 8, 2019 \\
Revised Aug 5, 2019 \\
Accepted Oct 6, 2019 \\
\hline
\end{tabular}

\section{Keywords:}

GPS module

IoT

Vehicle tracking system

\begin{abstract}
The quick development of innovation has made our lives less demanding. Innovation has additionally expanded activity risks and street mishaps occur very often which cause tremendous death toll and damage to property on account of poor response from the people in charge of managing such incidents. The mishap recognition undertaking will give an ideal solution for this problem. An accelerometer or a Tilt Sensor can be used as part of an auto caution application with the goal that unsafe driving can be identified. It can be utilized as a crash or rollover finder of the vehicle amid and after a crash. With signals from a sensor, a serious situation because of an accident can be avoided or attended to at the earliest. At the point of time when a vehicle meets with an accident or an auto moves over, the tilt sensor recognizes the flag and promptly sends it to the microcontroller. The microcontroller sends the alarm message through the IoT Module including the location of the accident through the GPS Module to the police or control group by publishing it over the cloud. So, the crisis enable group can promptly follow the area through the GPS Module, subsequent to receiving and accepting the data. The area can likewise be seen on the Google maps. Vital move can be made if this data reaches the control group in time. This venture is valuable in recognizing the accident with the use of sensors. As a future execution, we can add a remote webcam to the current system in order to capture pictures of the scene of the accident.
\end{abstract}

This is an open access article under the CC BY-SA license.

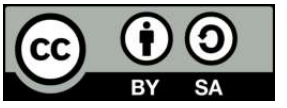

\section{Corresponding Author:}

Sudha Senthilkumar,

School of Information Technology and Engineering,

Vellore Institute of Technology,

Gorbachev Rd, Vellore, Tamil Nadu 632014, India.

Email: sudha.s@vit.ac.in

\section{INTRODUCTION}

The Internet of Things (IoT) is an arrangement of interrelated computing gadgets, mechanical and digital machines, objects, animals or individuals that are given one kind of an identifiers and the capacity to exchange information over a system without requiring human-to-human or human-to-PC communication. IOT is a new concept that has evolves from the convergence of wireless technologies. Wireless communication is the transfer of information or signal between two or more points that are not connected by an electrical conductor. IoT devices equipped with Wi-Fi allow the machine-to-machine communication. The sensor and actuator can be setup in different place but they are working together over an internet network [1].

Using IOT technique a vehicle tracking system (VTS) can be built. A vehicle tracking system combines the use of automatic vehicle location of individual vehicles with software that collects these fleet data for a comprehensive picture of vehicle locations. Modern vehicle tracking systems commonly use GPS technology for locating the vehicle, but other types of automatic vehicle location technology can also be used. Vehicle information can be viewed on electronic maps via internet with specialized software. 
The history of vehicle tracking dates to the beginning of GPS technology in 1978. In the early years, the technology was not yet operational, due to an insufficient number of satellites orbiting the earth. On Jan 17, 1994, after years of gradual growth, the final of the first 24 satellites was launched, and the GPS system was considered fully operational. Early GPS was designed primarily only for military but in 1996, President Bill Clinton determined that the system would be an asset to civilians as well as the military. This policy change made GPS technology available to the average individual, including fleet managers, who could see the benefit of using the technology to keep tabs on their vehicles. In the early days of fleet tracking, in order to properly track a fleet, each vehicle had to be enabled with a costly GPS device.

While helpful, these early systems were difficult to implement, costly to use and sometimes inconvenient for drivers and fleet management alike. Thus, it took several years for the concept to catch on. The modern fleet tracking system provides the necessary data to fleet managers allowing them to run their operations more efficiently. Reports on driver behavior, vehicle performance and fuel use all make it easier for the fleet manager to cut costs and increase efficiencies. These systems go beyond simple reporting of each vehicle's location, offering fleet managers a wealth of information about their vehicles and their drivers. In many countries this VTS is available. But there is no system which can detect accident and also give the service of VTS.

The proposed scheme considered to be a car safe project which can detect location of a car, and if there is any accident occur it can communicate automatically to the nearest police station, hospital and owner to reduce instant loss or damage. The quick development of innovation and framework has made our lives less demanding. The appearance of innovation has additionally expanded the activity risks and the street mishaps occur as often as possible which causes tremendous death toll and property on account of the poor crisis offices.

\section{OBJECTIVES}

The objective of this project is to detect an accident or a mishap at the earliest and inform the control groups in charge such as the police or hospitals so that they can react to this situation in minimum time possible and avoid any casualties and minimize damage to life and property by responding in a quick and efficient manner. This project is a combination of a VTS (Vehicle Tracking System) and finds out whether an accident has occurred or not and sends the message to the people in charge. The accident management data such as occurrence of the accident as well as the location of the accident are also published over a cloud and all the people that have access to the cloud server will have access to the data in order to provide the appropriate response measures.

\subsection{Overview of VTS}

Vehicle tracking Systems are now widely used in day to day life of human beings. The fundamental concept of the Vehicle Tracking Systems is based on the GPS technology. Nowadays, most of the cars, buses, trucks, ships and other automotive vehicles are fitted with GPS trackers. Vehicle Tracking and Information System, Automatic Vehicle Locating System, Mobile Asset Management System are commonly referred to as Vehicle Tracking Systems. These are widely used in developed countries for a variety of reasons. GPS technology serves multiple purposes however, the most important being tracking of vehicles and for navigation purposed. Tracking systems enable a base or headquarter to monitor the location of the vehicle and keep track of the driver remotely without the intervention of the driver. Tracking systems were first developed for the shipping industry because they wanted to determine where each vehicle was at any given time. Passive systems were developed in the beginning to fulfil these requirements. For the applications which require real time location information of the vehicle these systems can't be employed because they save the location information in the internal storage and location information can only be accessed when vehicle is available. To achieve Automatic vehicle location system that can transmit the location information in real time, active systems were developed. Real time vehicular tracking system incorporates a hardware device installed in the vehicle and a remote tracking server. The information is transmitted to the Tracking server using a Cloud protocol. This information is available to the users over the Internet. In active systems, users can get the real-time vehicle location on a Mobile Application with its current latitudinal and longitudinal data. This information is sent to the application through the MQTT protocol.

\subsection{Accident identification and alerting}

The frequency of occurrence of accidents have increased exponentially over the past few years. Every year a large number of people suffer immense damages to themselves as well as others when they are involved in an accident. There is catastrophic collateral damage as well as many people end up dying in 
accidents. The main reason for this is that accidents are not identified effectively and the people that have been injured in the accident are not given the medical attention that they require. Accident alerting systems are basically systems that are able to correctly identify when the accident has occurred with the help of sensors that are present in the car at the time of the accident. Multiple sensors such as shock sensors, temperature sensors, tilt sensors etc can be used for the purpose of accident identification. Each of these sensors can be programmed with values that full under the acceptable and range and values that signify the occurrence of an accident. For eg, if the accident identification system is using a tile sensor, the tilt sensor could be having a value greater than 100 . This means that the vehicle is currently in an upright and natural position and no accident has occurred. However, if the tilt sensor obtains a value of less than 100, it means that sensor has been moved from its original position and that an accident has occurred.Alerting systems are the extension of identification systems. Once an accident has occurred and the sensors have triggered an unacceptable value, this situation is then notified to the people in charge so that necessary action can be taken before there are fatal consequences. GSM has been widely used for the alerting portion as it is a very famous technology. However, we will be using a cloud protocol that sends a notification over the cloud to a mobile application which will intimate friends, family and control groups so that they can respond to the accident as soon as possible.

\section{LITERATURE SURVEY}

\subsection{Previous design ideas}

Previously we had accident alert systems using GSM and GPS modem and Raspberry Pi. A piezoelectric sensor first senses the occurrence of an accident and gives its output to the microcontroller. The GPS detects the latitude and longitudinal position of a vehicle. The latitudes and longitude position of the vehicle is sent as message through the GSM. The static IP address of central emergency dispatch server is pre-saved in the EEPROM. Whenever an accident has occurred the position is detected and a message has been sent to the pre-saved static IP address (NodeMCU, 2018).

Manasi Patil et al., described a better traffic management system using Raspberry pi and RFID technology. The vehicle has a raspberry pi controller fixed in it which is interfaced with sensors like gas sensor, temperature sensor and shock sensor. These sensors are fixed at a predetermined value before accident. When an accident occurs the value of one of the sensor changes and a message to a predefined number (of the ambulance) is sent through GSM. The GPS module which is also interfaced with the controller also sends the location of the vehicle. When the message is received by the ambulance, a clear route has to be provided to the ambulance. The ambulance has a controller ARM which is interfaced with the RFID tag sends electromagnetic waves. When an ambulance reaches the traffic signal the RFID reader which is placed on the joints detect the electromagnetic waves of the tag. If the traffic signal is red, then the readers goes through the database in fraction of seconds and turn the red-light green. And automatically in such condition the RFID on opposite joints turn the opposite signal red. This provides a clear route to the ambulance. Apurva Mane et al., described the methods for vehicle collision detection and remote alarm device using Arduino. Key features of this design include real-time vehicle monitoring by sending its information regarding position (longitude, latitude), time, angle to the monitoring station and to the user/owners mobile that should help them to get medical help if accident or the theft occurs. Also, user/owner has an access to get real-time position of a vehicle in real time. Whenever accident occurs MEMS and vibration sensor detects and sends the signals to microcontroller, by using GPS particular locations where accident has occurred is found, then GSM sends message to authorized members. Bhagya Lakshmi, proposed a FPGA Based Vehicle Tracking and Accident Warning system using GPS. FPGA is mainly used to track position of any vehicle and send automated message to pre-programmed number. The owner of vehicle, police to clear traffic, ambulance to save people can be informed by this device. FPGA controls and coordinate all parts used in system. With the help of accelerometer sensor, the exact position of the vehicle can be detected. It can also be predicted whether the vehicle is in normal position or upside down.

The systems that have been mentioned above rely heavily on different types of sensors and different technologies that may not be readily available. Such systems have very high initial investments and have high maintenance and installation costs. Due to the high initial investments companies and people don't opt for such technologies and prefer cheaper alternatives which are not as efficient as the systems that are mentioned above. However, matters such as these involving human lives and huge collateral damage need to be efficient and easy to maintain while being reliable. The proposed setup of the project does not involve the usage of multiple sensors, due to which the installation costs are relatively low. Also, the parts used in the proposed setup are readily available and replaceable in case of additional parts are required. The proposed setup of the project involves the usage of Tilt sensors or accelerometer to send a signal to the IoT device which includes the WiFi module. The GPS module that is attached allows us to track the location of the

Vehicle accident management and control system using MQTT (Sudha Senthilkumar) 
vehicle and provides the latitude and longitudinal data of the location of the accident. This data is then sent to the cloud using MQTT protocol and can be accessed by control groups or hospitals so that the people in charge can respond to the situation in minimum time and prevent loss of life and reduce the amount of damage caused. Figure 1 and Figure 2 show the block diagram and flowchart of the system. The connection of the component is shown in Figure 3.

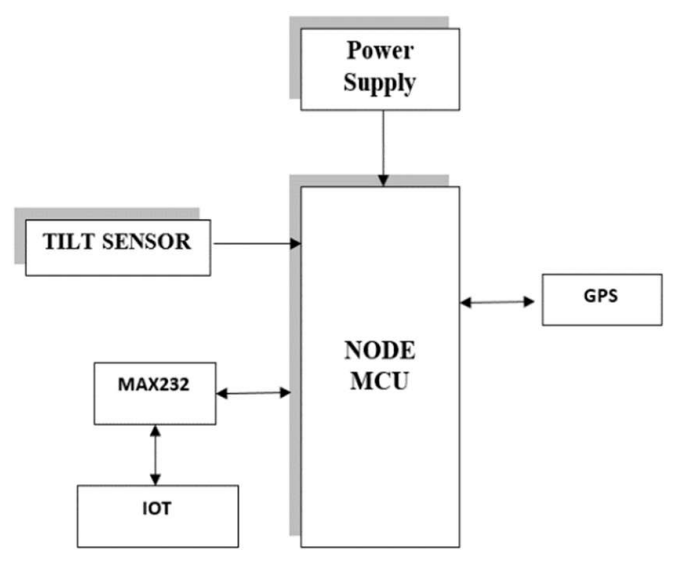

Figure 1. Block diagram of system

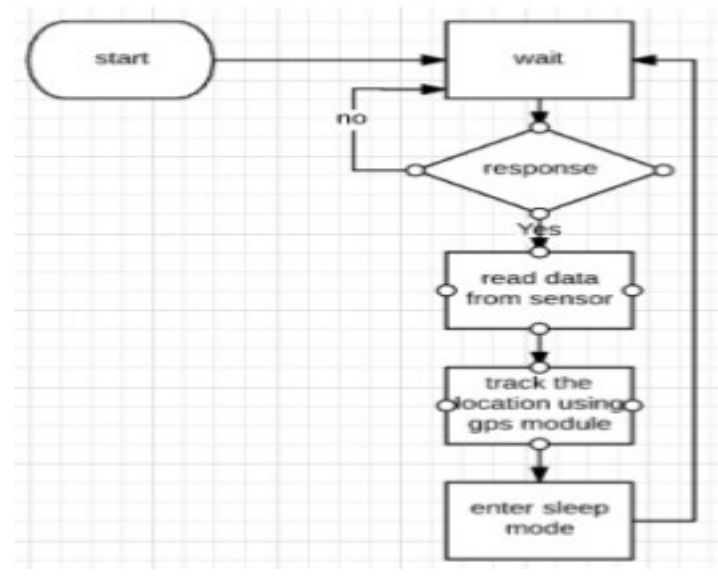

Figure 2. Flowchart of the system

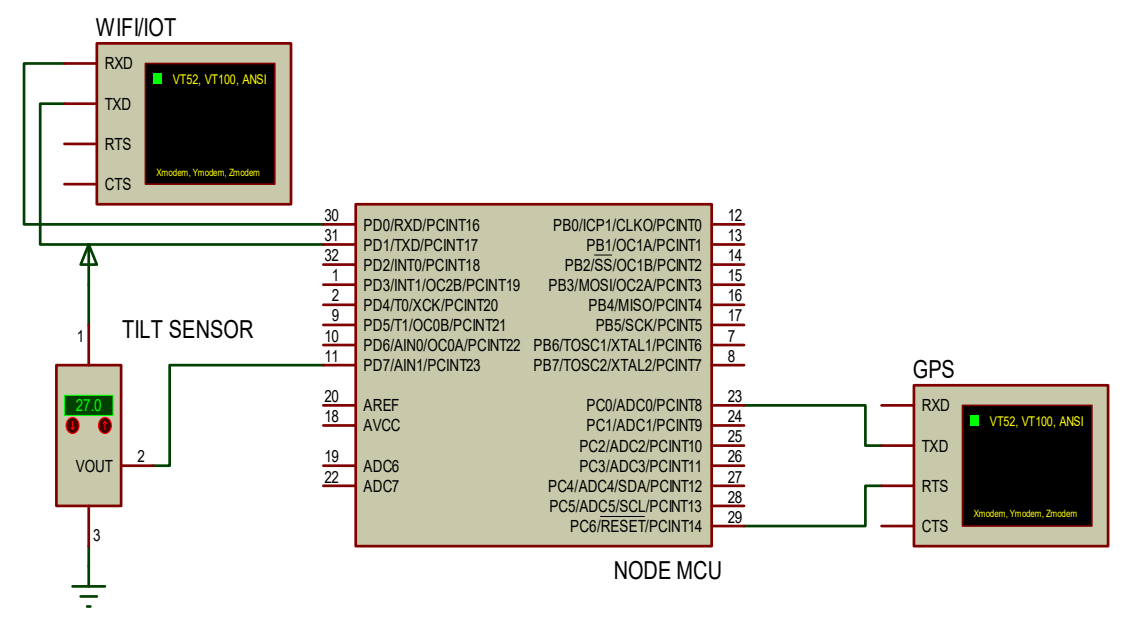

Figure 3. Connection of the components

\section{VARIOUS MODULE DESIGN}

\subsection{NodeMCU}

The NodeMCU is an open-source firmware and development kit that helps you to prototype your IoT product with few Lua script lines. The Development Kit based on ESP8266, integrates GPIO, PWM, IIC, 1-Wire and ADC all in one board. The ESP8266 is the name of a micro controller designed by Espressif Systems. The ESP8266 itself is a self-contained WiFi networking solution offering as a bridge from existing micro controller to WiFi and is also capable of running self-contained applications. This module comes with a built in USB connector and a rich assortment of pin-outs. With a micro USB cable, you can connect NodeMCU devkit to your laptop and flash it without any trouble, just like Arduino as shown in Figure 4. It is also immediately breadboard friendly. The MQTT library has been ported to the ESP8266 SoC platform and committed to the NodeMCU project. After this, the NodeMCU was able to support the MQTT IoT protocol using Lua to access the MQTT broker [1]. Figure 5 shows the pin diagram of NodeMCU. 

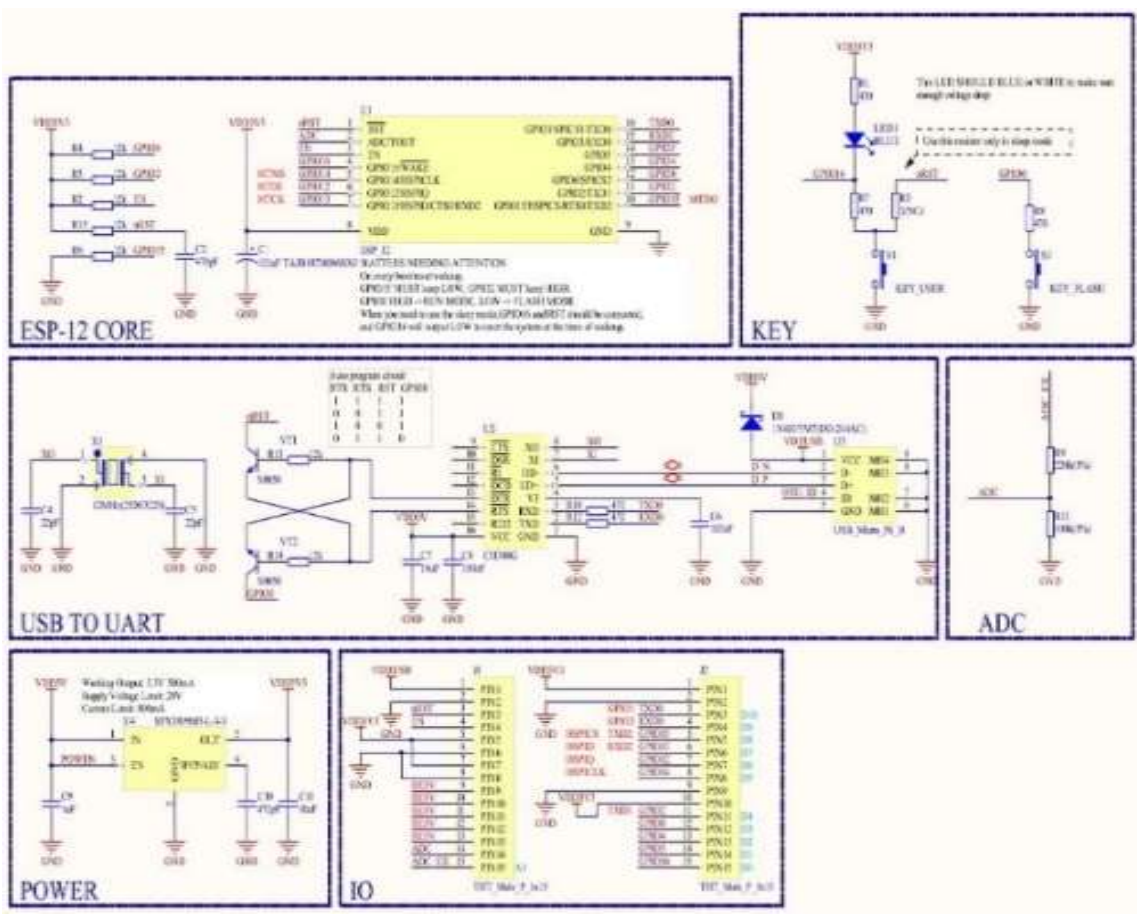

Figure 4. Schematic diagram of NodeMCU devkit

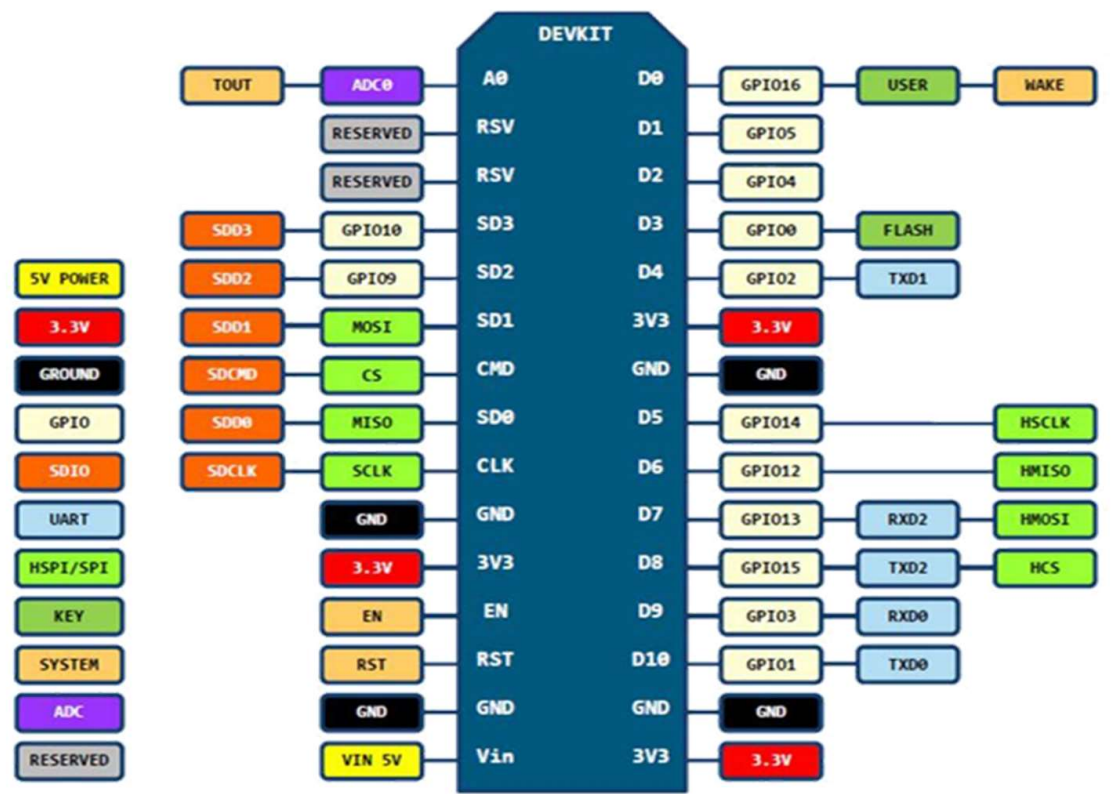

De(GPIO16) can only be used as gpio read/write, no interrupt supported, no pwm/izc/ow supported.

Figure 5. Pin diagram of NodeMCU

\subsection{ESP8266 Wi-Fi unit}

The ESP8266 WiFi Module is a self-contained SOC with integrated TCP/IP protocol stack that can give any microcontroller access to your WiFi network. The ESP8266 is capable of either hosting an application or offloading all Wi-Fi networking functions from another application processor. Each ESP8266 module comes pre-programmed with an AT command set firmware, meaning, you can simply hook this up to 
your Arduino device and get about as much Wi-Fi-ability as a Wi-Fi Shield offers. The ESP8266 module is an extremely cost-effective board with a huge, and ever growing, community. This module has a powerful enough on-board processing and storage capability that allows it to be integrated with the sensors and other application specific devices through its GPIOs with minimal development up-front and minimal loading during runtime. Its high degree of on-chip integration allows for minimal external circuitry, including the front-end module, is designed to occupy minimal PCB area. The ESP8266 supports APSD for VoIP applications and Bluetooth co-existence interfaces, it contains a self-calibrated RF allowing it to work under all operating conditions, and requires no external RF parts [2]. It also has the capabilities of a microcontroller, very similar to Arduino and can be programmed using the Arduino IDE. Arduino IDE has an extension specifically for the ESP8266 so that it can be programmed with relative ease [2]. Figure 6 illustrates the ESP8266 pin diagram.

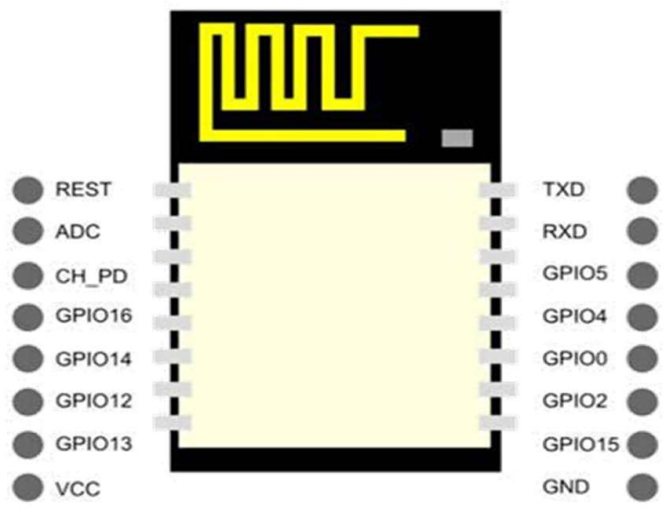

Figure 6. ESP8266 pin diagram

\subsection{GPS module}

Global Positional System or GPS uses a Global Navigation Satellite System made up of a network of many satellites placed into the orbit by the US Dept. of Defense. A GPS Navigation Device or simply GPS is a device that is capable of receiving information from the GPS satellites in order to calculate the device's geographical position. The GPS device receives this information from the satellites in terms of coordinates or latitudes and longitudes. Usage of appropriate software can allow us to plot those coordinates or latitudinal and longitudinal data on a map for easier and clearer understanding.

Nowadays, most of the GPS modules are used in vehicles like cars, bikes, buses, trucks etc. Smartphones with GPS capability use (A-GPS) or Assisted GPS technology, which generally use the base station or towers to provide the tracking capability of the device. However, when we are outside the network range, the A-GPS will not be available for use as it requires the device to be in an area with network coverage. Since the GPS Module is connected with satellites, despite an area not having network connectivity, the GPS module will continue to function and provide the coordinates or the position of the system. A GPS device can retrieve from the GPS system location and time information in all weather conditions, anywhere on or near the Earth. A GPS reception requires an unobstructed line of sight to four or more GPS satellites, and is subject to poor satellite signal conditions. In exceptionally poor signal conditions, for example in urban areas, satellite signals may exhibit multipath propagation where signals bounce off structures, or are weakened by meteorological conditions. Obstructed lines of sight may arise from a tree canopy or inside a structure, such as in a building, garage or tunnel [3]. The pin diagram of GPS module is shown in Figure 7.

Int J Adv Appl Sci, Vol. 9, No. 1, March 2020: 1 - 11 




Figure 7. Pin diagram of GPS module

\subsection{Tilt sensor}

Acceleration is a measure of how quickly speed changes. Just as a speedometer is a meter that measures speed, an accelerometer is a meter that measures acceleration. We can use the accelerometer's ability to sense acceleration to measure a variety of things that are useful to electronic and robotic projects and designs such as: Acceleration, Tilt Angle, Incline, Rotation, Vibration ,Collision, Gravity [4]. For the purpose of our system we are using an accelerometer as our Tilt sensor which will be used to detect the occurrence of an accident or not.

\subsection{MQTT}

MQTT is a machine-to-machine/ "Internet of Things" publisher-subscriber based connectivity protocol. It was designed as an extremely lightweight publish/subscribe messaging transport. It is useful for connections with remote locations where a small code footprint is required and/or network bandwidth is extremely expensive. Message Queueing Telemetry Transport is an ISO standard publish-subscribe based messaging protocol. It works on top of the TCP/IP protocol. The publish-subscribe based messaging pattern requires a message broker.

Message brokers are fundamentally programs that act as middleman between the sender and the receiver. It is the responsibility of the message broker to convert the message from the messaging protocol of the sender to the appropriate messaging protocol of the receiver. Message brokers are commonly used in computer networks or telecommunication where multiple software applications need to interact with one another by sending formally-defined messages.For the purpose of our system we are using the Mosquitto message broker which is lightweight and suitable for all devices from low power single board computers to full servers.

The MQTT protocol provides a lightweight method of carrying out messaging using a publish/subscribe model. This makes it suitable for Internet of Things messaging such as with low power sensors or mobile devices such as phones, embedded computers or microcontrollers. The MQTT protocol is a good choice for wireless networks that experience varying levels of latency due to occasional bandwidth constraints or unreliable connections. Should the connection from a subscribing client to a broker get broken, the broker will buffer messages and push them out to the subscriber when it is back online. Should the connection from the publishing client to the broker be disconnected without notice, the broker can close the connection and send subscribers a cached message with instructions from the publisher [5].

\section{IOT, ALGORITHM ,TEST CASES}

\subsection{Internet of Things (IoT)}

The internet of things (IoT) is the network of physical devices, vehicles, buildings and other items embedded with electronics, software, sensors, actuators, and network connectivity that enable these objects to collect and exchange data. The IoT allows objects to be sensed and controlled remotely across existing network infrastructure, creating opportunities for more direct integration of the physical world into computer-

Vehicle accident management and control system using MQTT (Sudha Senthilkumar) 
based systems, and resulting in improved efficiency, accuracy and economic benefit. When IoT is augmented with sensors and actuators, the technology becomes an instance of the more general class of cyber-physical systems, which also encompasses technologies such as smart grids, smart homes, intelligent transportation and smart cities. Each thing is uniquely identifiable through its embedded computing system but is able to interoperate within the existing Internet infrastructure. Experts estimate that the IoT will consist of almost 50 billion objects by 2020 [6].

IoT will become an essential part of the lives of humans impacting us in a very big way. It will also become one of our sustenance requirements such as Internet, telephone, water, electricity. IoT's impact will be similar to the impact of Internet at the time. However, Internet connected individual computers which could be at different locations whereas IoT will connect day-to-day usage objects which will have a strong impact in the physical world [6-8].

Data management is a crucial aspect in the Internet of Things. When considering a world of objects interconnected and constantly exchanging all types of information, the volume of the generated data and the processes involved in the handling of those data become critical. A long-term opportunity for wireless communications chip makers is the rise of Machine-to-Machine (M2M) computing, which one of the enabling technologies for Internet of Things [9, 10]. This technology spans abroad range of applications. While there is consensus that M2M is a promising pocket of growth, analyst estimates on the size of the opportunity diverge by a factor of four. Conservative estimates assume roughly 80 million to 90 million M2M units will be sold in 2014, whereas more optimistic projections forecast sales of 300 million units. Based on historical analyses of adoption curves for similar disruptive technologies, such as portable MP3 players and antilock braking systems for cars, it is believed that unit sales in M2M could rise by as much as a factor of ten over the next five years. There are many technologies and factors involved in the "data management" within the IoT context. Some of the most relevant concepts which enable us to understand the challenges and opportunities of data management are Data Collection and Analysis, Big Data, Semantic Sensor Networking, Virtual Sensors, Complex Event Processing [11-13].

\subsection{Algorithm}

Step 1: Start

Step 2: Power on all modules

Step 3: Wait for the tilt sensor to detect an accident

Step 4: Once accident is detected, location of the accident is sent from the GPS Module to the NodeMCU

Step 5: Send location as well as accident status to the cloud using MQTT protocol.

Step 6: Rescue groups having access to the cloud are given real-time notification.

Step 7: End

\subsection{Test cases}

- In order to check if the system is working correctly, we can perform a few test cases. If the system passes the test cases, we can safely say that the system is working as per the requirements.

- In order to check if individual components of the system are working we can perform the following the checks:

Test case 1: Tilt Sensor: When the tilt sensor is in a natural or acceptable position the led light on the sensor does not glow. However as soon as it's been tilted to a position which is unnatural or unacceptable, the led light glows. Therefore, we can check whether the tilt sensor is working correctly by tilting it at different angles and seeing whether the led starts glowing at angles above the threshold value.

Test case 2: GPS Module: The GPS Module is responsible for giving the latitudinal and longitudinal values based on the position of the current system. We can check whether the GPS Module is working correctly if we are able to see the latitudinal and longitudinal values and checking it those values correspond to our current position. If the values are being displayed as 0,0 for latitude and longitude, it means that the GPS Module is not functioning as desired.

Test case 3: MQTT App: As soon as the Tilt sensor detects the change in angle of inclination, it sends the notification to the MQTT application in real-time. If we are unable to see the values and the notification on the application, it means that the MQTT protocol is not working properly or the system is not connected to the server. If the values are displayed properly with the notification, it means that the system is connected to the server successfully and values are being displayed for the use of the user.

Int J Adv Appl Sci, Vol. 9, No. 1, March 2020: 1 - 11 


\subsection{Implementation results}

After completing the test cases and making sure all the system components are fully functioning, we can start using the system. Once the system is implemented the following observations are made:

a. Once the system is in an unnatural position, the led light on the tilt sensor starts glowing which signifies that the tilt sensor is in the position when an accident has occurred.

The tilt sensor is used to detect the change in the g-forces in the object. The change in g-forces indicates the status of collision or crash of the object. Accelerometers are used for measuring vehicle body movement in various systems such as advanced braking system, passive restraint and electronically controlled suspension systems, etc. The tilt sensor has a more sensitivity and high stable in automotive environment. The tilt sensor module is essential module for vehicle accident detection system. It is used as automatic ECS (Emergency Calling System, which senses the change in the g-forces of the system. Actual thresholds g-forces for accident detection [14] are shown in Table 1.

Table 1. Thresholds g-forces for accident detection [11]

\begin{tabular}{cc}
\hline Accident harshness & Actual Maximum G Range representation \\
\hline No Accident & $0-4 \mathrm{~g}$ \\
Mild Accident & $4-20 \mathrm{~g}$ \\
Medium Accident & $20-40 \mathrm{~g}$ \\
Severe Accident & More than $40 \mathrm{~g}$ \\
\hline
\end{tabular}

Due to the impact of the earth's gravitational forces, change in the vehicle attitude will result in normal accelerations. The sensitivity of the tilt sensor is measured in the terms of the g-forces which are measured at the time of crash. The accident is detected by presence of vibration in the vehicle at the time of crash and the threshold value of the sensitivity can be measured. The g-forces of vehicles during crash are shown in Table 2. The location of the accident is intimated along with integration of google Map is added advantage in the proposed system which is not exist in the previous systems which is discussed [14, 15].

Table 2. Vehicle G-forces during crash

\begin{tabular}{ccccc}
\hline S. No & g-forces & Severity of Accident & Tilt sensor & Message Activated \\
\hline 1 & $4 \mathrm{~g}$ & No Accident & Sensor off & No \\
2 & $10 \mathrm{~g}$ & Mild Accident & Sensor off & No \\
3 & $14 \mathrm{~g}$ & Mild Accident & Sensor off & No \\
4 & $40 \mathrm{~g}$ & Major Accident & Sensor Glow & App Message Activated, Location is given \\
5 & $60 \mathrm{~g}$ & Major Accident & Sensor Glow & App Message Activated, location is given. \\
6 & $70 \mathrm{~g}$ & Major Accident & Sensor Glow & App Message Activated, location is given. \\
7 & $73 \mathrm{~g}$ & Major Accident & Sensor Glow & App message Activated, location is given. \\
\hline
\end{tabular}

b. The MQTT Application displays 2 variables, one displays a notification whether an accident has occurred or not as shown in Figure 8 and Figure 9 respectively. "ACCIDENT OCCURRED" is displayed when the tilt sensor is tilted at an angle that is beyond the set threshold. "NO ACCIDENT" is displayed when the tilt sensor is in its natural position as shown in Figure 10. 


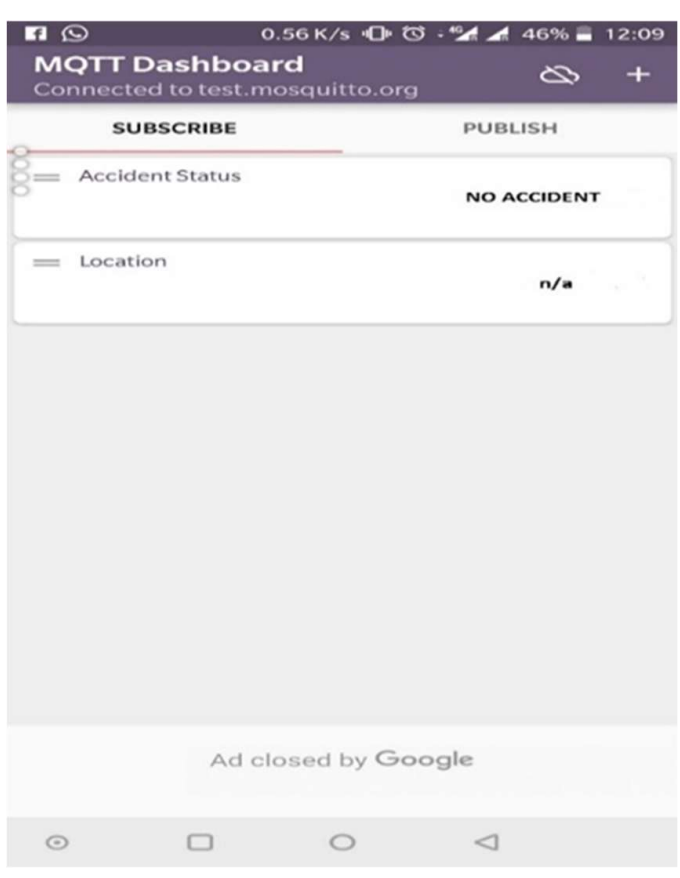

Figure 8. Output of MQTT app when tilt sensor is in natural position

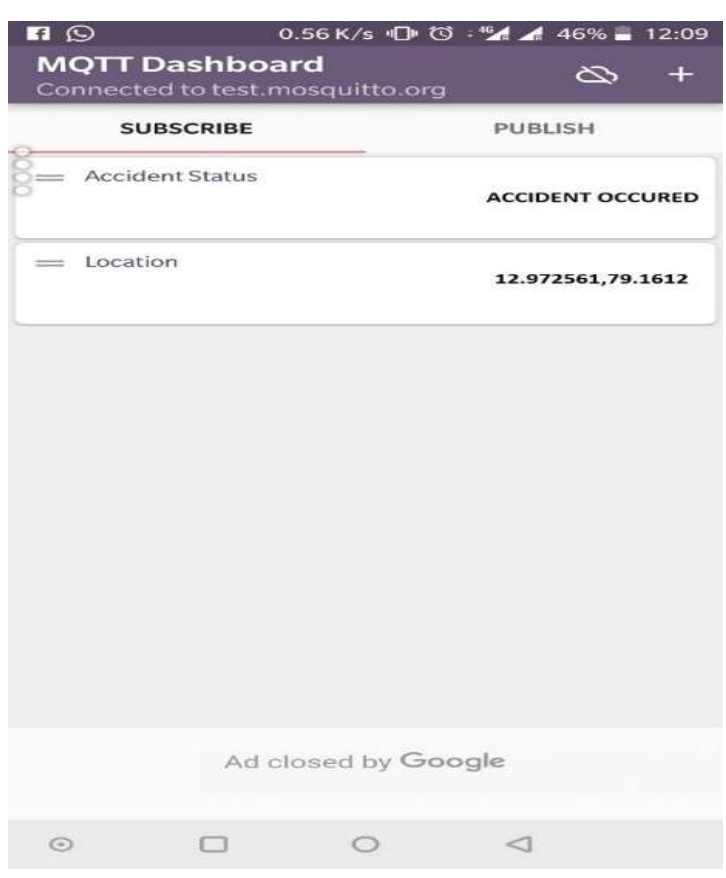

Figure 9. Output of MQTT app when an accident has occurred

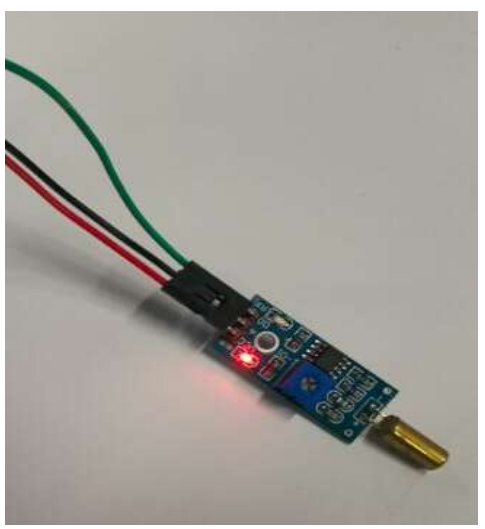

Figure 10. Tilt sensor led glow

\section{CONCLUSION AND FUTURE SCOPE}

Hence the automatic alarm device for vehicle accidents has been implemented using NODE MCU microcontroller. This design is a system which can detect accidents in significantly less time and sends the basic information to first aid center within a few seconds covering geographical coordinates, the time in which a vehicle accident has occurred.

Although the system is fully functional, some enhancements can be made in and around the system in order to make it even better and overcome some of the drawbacks of the existing system. A camera can be attached to the system in the vehicle so that the camera can click pictures at the time of the accident which may help the doctors in identifying the extent of injury of the people in the car and give the appropriate treatment. Also, the pictures will help the police in understanding the sequence of events and take action against the people responsible for the accident. A switch can be added to prevent false alarms or if the accident is not as severe and the driver and others are able to take care of themselves without needing urgent and immediate support. 


\section{REFERENCES}

[1] Apurva Mane and Jaideep Rana, "Vehicle Collision detection and Remote Alarm Device using Arduino," International Journal of Current Engineering and Technology, Vol. 4, No. 3, June 2014.

[2] ESP8266 (n.d.). Retrieved March 27, 2018, [Online]. Available: https://en.wikipedia.org/wiki/ESP8266

[3] GPS Module. Retrieved March 27, 2018, [Online]. Available: https://en.wikipedia.org/wiki/ GPS_navigation_device

[4] Kiran Sawant, Imran Bhole, Prashant Kokane, Piraji Doiphode, and Yogesh Thorat, "Accident Alert and Vehicle Tracking System," International Journal of Innovative Research in Computer and Communication Engineering, Vol. 4, No. 5, May 2016.

[5] MQTT. Retrieved March 27, 2018, [Online]. Available: https:// en.wikipedia.org/wiki/MQTT

[6] Internet of Things. Retrieved March 27, 2018, [Online]. Available: https://en.wikipedia.org/wiki/Internet_of_things

[7] William M. Evanco, "The Impact of Rapid Incident Detection on Freeway Accident Fatalities," MS - IVHS, 1996.

[8] Statistics of Accidents in India, 2008. [Online]. Available: http://www.nitawriter.wordpress.com/2008/

[9] David A. Whitney and Joseph J Pisano TASC, Inc., Reading, Massachusetts, "Auto Alert: Automated Acoustic Detection of Incidents," IDEA project, 1995.

[10] Manasi Patil, Aanchal Rawat, Prateek Singh, and Srishtie Dixit, "Accident Detection and Ambulance Control using Intelligent Traffic Control System," International Journal of Engineering Trends and Technology (IJETT), Vol. 34, No. 8, April 2016.

[11] NodeMCU. Retrieved March 27, 2018, [Online]. Available: https://en.wikipedia.org/wiki/NodeMCU

[12] Sri Krishna Chaitanya Varma, Poornesh, Tarun Varma, and Harsha, "Automatic Vehicle Accident Detection and Messaging System Using GPS and GSM Modems," International Journal of Scientific \& Engineering Research, Vol. 4, No. 8, August 2013.

[13] V.Sagar Reddy, L. Padma Sree, and V. Naveen Kumar, "Design and Development of accelerometer-based System for driver safety," International Journal of Science, Engineering and Technology Research (IJSETR), Vol. 3, No. 12, December 2014.

[14] EEE Technical Committee for Sensor Technology, October 2009. The IEEE P1451.6 Project. [Online]. Available: http://grouper.ieee.org/groups/1451/6/index.htm

[15] D. Punetha, D. Kumar, and V. Mehta, "Design and realization of the Accelerometer based Transportation System (ATS)," International Journal of Computer Applications, Vol. 49, No. 15, 2012. 